\title{
Functional Magnetic Resonance Imaging of Compensatory Neural Recruitment in Aging and Risk for Alzheimer's Disease: Review and Recommendations
}

\author{
S. Duke Han ${ }^{a-c}$ Katherine J. Bangen ${ }^{d}$ Mark W. Bondie, ${ }^{f}$ \\ aDepartment of Psychology, Loyola University Chicago, Chicago, III., b Department of Neurology and \\ 'Neuroscience Institute, Loyola University Medical Center, Maywood, III., dSDSU/UCSD Joint Doctoral Program, \\ e Department of Psychiatry, University of California San Diego School of Medicine, and ${ }^{\mathrm{f}}$ Psychology Service, \\ VA San Diego Healthcare System, San Diego, Calif., USA
}

\section{Key Words}

Aging - Alzheimer's disease - APOE - Compensation •

Dedifferentiation $\cdot$ Dementia $\cdot$ Functional magnetic

resonance imaging $\cdot$ Functional neuroimaging •

Region-Activation-Performance model (RAP model) ported. This proposed model addresses significant brain region, activation pattern, and behavioral performance considerations, and is therefore termed the Region-ActivationPerformance model (RAP model).

Copyright $\odot 2008$ S. Karger AG, Basel

\section{Introduction}

Neuroimaging studies of normal aging, genetic risk for Alzheimer's disease (AD), and mild cognitive impairment (MCI) have reported between-group differences in functional neuroimaging response patterns or metabolic changes during cognitive tasks - often in the context of equivalent behavioral performance [1]. Such findings have routinely been interpreted as evidence for compensatory neural recruitment in which older adults employ additional brain regions in order to maintain a certain level of behavioral performance. In fact, a majority of studies in this area of functional neuroimaging research have invoked compensatory recruitment to explain the patterns of results between groups.

Indeed, neuroimaging results are often interpreted as evidence for compensatory recruitment despite factors that may suggest alternative explanations such as dedif-

\section{KARGER}

() 2008 S. Karger AG, Base

Fax +41613061234 E-Mail karger@karger.ch www.karger.com www.karger.com/dem
S. Duke Han, PhD

Department of Psychology

6525 N. Sheridan Road

Chicago, IL 60626 (USA)

Tel. +1 773508 3073, Fax +1 773508 8713, E-Mail dhan2@luc.edu 
ferentiation of cognitive abilities [for a review, see ref. 2] or greater neural noise [3]. Thus, the need is great for standardized criteria for interpreting functional neuroimaging findings as evidence for compensation. In this critical review, we survey a selection of the aging and $\mathrm{AD}$ risk literature that has contributed to the development of the concept of compensatory neural recruitment from a neuroimaging perspective, and we conclude with a proposed set of criteria to account for brain region, activation, and performance (RAP) considerations when interpreting observed functional magnetic resonance imaging (fMRI) results as compensatory.

\section{Normal Aging}

Normal aging is associated with declines in cognitive domains including memory, reasoning and spatial abilities [4]. Functional neuroimaging studies of nondemented older adults have reported a variety of findings including decreased brain response, increased brain response and decreases in typical lateralization [e.g. ref. 5]. Two main theories have been proposed to explain decreased and increased brain activation in older adults: the functional compensation theory and the dedifferentiation theory [2]. According to the compensation view, decreased activation in deep gray matter structures in older relative to young participants may indicate less efficient processing. In contrast, increased activity is often taken to reflect compensatory mechanisms indicative of reallocation of neural resources, reorganization of function such that new regions take on the tasks of declining regions [e.g. ref. 6], or recruitment of different strategies or processes [e.g. ref. 5] to maintain or support performance.

Support for the compensation view has come from neuroimaging studies assessing the relation between brain activity and cognitive performance [7]. Several studies have reported an association between increased brain activation and better cognitive performance. For instance, one group reported that older adults who demonstrated bilateral activity were faster on a verbal working memory task [8]. Corroborating such findings, Cabeza et al. [9] conducted a positron emission tomography (PET) study of recall and source memory and found that lowperforming older adults demonstrated right prefrontal cortex (PFC) activation similar to young adults. Highperforming older adults, however, displayed bilateral PFC activation. Cabeza et al. [9] argued that the observed hemispheric asymmetry reduction in high-performing older adults was consistent with the compensation view and inconsistent with the dedifferentiation view. The authors further concluded that low-performing older adults engaged neural networks similar to those recruited by young adults, but used them less efficiently, whereas highperforming older adults recruited reorganized networks as a means of compensating for age-related decline.

In addition to the two findings discussed above, increased bilaterality of activity in older adults is often associated with enhanced cognitive performance, and brain regions showing additional bilateral activity in older adults are presumed to enhance performance on the investigated task. Based on functional neuroimaging findings, Cabeza [7] introduced this as a cognitive neuroscience model called 'hemispheric asymmetry reduction in older adults' (HAROLD). Providing empirical support for the HAROLD model and controlling for betweengroup differences in cognitive ability, Anderson et al. [10] conducted a blood-oxygenated level-dependent (BOLD) fMRI study in which cognitively intact, normal older adult participants performed a word recognition task involving an easy condition and a difficult condition that was titrated to each participant's ability. In the latter condition, the list length was determined prior to scanning in a training session and varied so that each participant achieved $75 \%$ performance accuracy. Using this method, the authors ensured that all participants were equally challenged and controlled for individual differences in memory abilities. During the difficult condition (relative to easy condition), participants demonstrated increased activation of the left medial frontal, right superior temporal, and right superior parietal regions. Anderson et al. [10] concluded that increased activation of bilateral regions during the difficult condition may reflect recruitment of additional regions in order to maintain performance in light of increased attention and monitoring demands. The bilateral activation on the word recognition task may reflect compensatory recruitment of right hemisphere neural networks. Many fMRI studies do not incorporate methods to control for task difficulty and ensure that participants are equally challenged and, therefore, the task titration technique used in this study represents a methodological strength and relatively unique feature.

Grady et al. [11] originally proposed that older adults might engage strategies mediated by PFC in order to compensate for deficits in sensory processing mediated by occipital cortex. Furthermore, Dennis and Cabeza [referenced as forthcoming in ref. 12] called this consistent finding a 'posterior-anterior shift in aging' (PASA) 
and conducted a study to determine whether PASA is independent of cognitive task and difficulty level. In the study, participants completed an episodic retrieval task (word recognition) and visual perception task (area judgment). Findings included age-related decreases in occipital activity accompanied by increases in PFC activity as well as an association between increased PFC activation and better cognitive performance, suggesting that PASA is generalizable to different types of cognitive tasks. As Davis et al. [12] suggest, brain activation varies as a function of task difficulty, and the same tasks are often more challenging for older relative to younger adults. Therefore, increased activation could be related to increased difficulty rather than aging per se. In order to eliminate differences in difficulty, Davis et al. [12] matched young and older adults on performance accuracy on the task and still found engagement patterns consistent with the PASA model. Thus, PASA differences appear to be related to age-related processes rather than task difficulty, and the findings support the compensatory role of PASA.

In contrast to the compensatory view, the dedifferentiation view reflects greater engagement of generalized processes [13]. The dedifferentiation view is based, in part, on the replicable finding in psychometric research involving positive correlations among measures assessing various cognitive abilities and on cross-sectional studies of intelligence reporting a general pattern involving lack of differentiation during childhood, differentiation of abilities in adulthood, and dedifferentiation in late adulthood [2]. According to Li and Lindenberger [2], evidence for the dedifferentiation view comes from behavioral studies reporting stronger intercorrelations among tasks that assess the same cognitive domain as well as across different abilities in older adults relative to young adults. In addition, Li and Lindenberger [2] cite evidence from neuroimaging studies that suggest a greater level of interdependence among various processes in older adults. For instance, they describe work by Grady et al. [11] reporting greater activation in the PFC during object matching and location matching in older adults relative to young participants. Grady et al. [11] argued that during visual processing, young adults use circuitry in the occipital lobe more efficiently than older adults and that older adults showed more 'reliance' on additional regions, suggesting a greater level of interdependence among various functions at the cortical level. Furthermore, Li et al. [14] propose a 'theoretical link' in which age-related deficiencies in neuromodulation lead to decreased responsiveness of neurons and increased neural noise, which may lead to dedifferentiation of cortical representations.
In contrast to the HAROLD model, the dedifferentiation view suggests that decreases in hemispheric asymmetry are simply 'byproducts of aging' [9] and do not have a specific function. Further, there is empirical evidence inconsistent with the HAROLD model. In at least one study, PFC activation was bilateral in young participants but unilateral in older adults [15]. In addition, a recent fMRI study [16] found that improved performance of a task was associated with increased hemispheric asymmetry in PFC in older adults. These authors argued that their findings were inconsistent with the compensatory hypothesis but consistent with a 'complementary' view [17] in which improved task performance is not necessarily associated with decreased asymmetry, but depends on the role of the active regions in the particular task.

As Cabeza [18] notes, the compensation and dedifferentiation views are not incompatible. For instance, Cabeza [18] suggests that combining cognitive functions could counteract cognitive decline associated with aging. In addition, there is a lack of definitive evidence indicating that age-related increases in correlations among cognitive abilities have detrimental effects on cognition. Cabeza [18] also argues that decreased hemispheric asymmetry may serve a compensatory role and lead to more similarity across various tasks and, thus, could account for the increases in correlations across cognitive measures seen in aging. Furthermore, as Beason-Held et al. [13] suggest, there are possible explanations for increases in age-related activation in addition to the compensation and dedifferentiation views, and these include the presence of greater neural noise [20].

\section{Genetic Risk}

Several neuroimaging studies have provided evidence that the HAROLD model may be generalizable to individuals at genetic risk for Alzheimer's disease, as defined by the presence of at least one APOE $\varepsilon 4$ allele. In perhaps the original study, Bookheimer et al. [21] compared performance and brain response between APOE $\varepsilon 4$ carriers and a group that was homozygous for the $\varepsilon 3$ allele in a challenging memory task involving listening to unrelated pairs of words (learning blocks) and then being presented with the first word in each pair and silently recalling the second word (recall blocks). The APOE $\varepsilon 4$ carriers demonstrated greater magnitude and extent of activation in regions including the left hippocampal, parietal, and prefrontal regions. Longitudinal findings indicated that the 
degree of baseline brain activity was associated with memory decline two years later. The authors reported greater brain activation differences between the groups during recall, in which participants had to put forth cognitive effort in order to retrieve the stimuli, relative to learning trials, in which participants listened to words but did not actively recall information. In addition, among the APOE $\varepsilon 4$ carriers, increased activation was observed in the anterior cingulate and dorsal PFC, which are areas that demonstrate increased activation as cognitive effort increases [22]. Bookheimer et al. [21] suggested that one explanation for the observed pattern of brain activation is that $\varepsilon 4$ carriers require additional cognitive resources in order to achieve and maintain a performance level similar to the non- $\varepsilon 4$ carriers. The authors proposed that the $\varepsilon 4$ carriers demonstrate changes in brain function prior to the appearance of observable memory decline or neuroanatomical alterations.

Bondi et al. [23] compared performance and brain response to a landscape picture encoding task between individuals at genetic risk for $\mathrm{AD}$ and those not at risk. APOE $\varepsilon 4$ carriers demonstrated greater brain response during novel picture encoding in bilateral fusiform gyrus, right superior parietal lobule, left middle frontal gyrus, and medial frontal gyrus relative to individuals who were homologous for the $\varepsilon 3$ allele. Given that the two groups both had normal learning and memory capabilities, comparable gray, white, and cerebrospinal fluid volumes, and no baseline differences in the control condition, and that there were no broad differences in physiology based on the fact that both groups demonstrated a strong positive bilateral response to the encoding condition in primary visual cortex, differences appear to be more directly related to an individual's genotype. Findings are consistent with a compensatory hypothesis in which the $\varepsilon 4$ individuals demonstrate greater magnitude and extent of activation in order to achieve the same level of performance. Further supporting this view, we [1] recently reported that APOE $\varepsilon 4$ carriers demonstrated greater activity in several right hemisphere regions relative to non- $\varepsilon 4$ carriers during a verbal paired-associate task. We concluded that additional frontal and temporal activation provides evidence for the recruitment of executive functions and semantic memory processes to compensate for deficits in episodic memory encoding associated with $\varepsilon 4$ allele.

To determine whether increased activation in at-risk individuals is specific to memory encoding or generalizable to other cognitive abilities, Burggren et al. [24] studied at-risk older adults using a digit span task. Regardless of genetic status, increasing difficulty, defined as increasing digit length, was associated with greater magnitude and extent of PFC activation. Burggren et al. concluded that increased brain activation was associated with increased cognitive demand. In addition, they proposed that greater brain activation in at-risk individuals may be specific to memory tasks and not generalizable to other domains.

\section{Mild Cognitive Impairment}

Dickerson et al. [25] reported that individuals with MCI show better memory performance on a postscanning recognition test; this was associated with greater parahippocampal activation. Interestingly, MCI individuals with greater impairment demonstrated greater parahippocampal engagement during encoding, indicating that greater MTL activation may represent a compensatory response to increasing $\mathrm{AD}$ pathology. Corroborating the findings by Dickerson et al. [25], a recent study [26] reported increased hippocampal activation in MCI compared to normal controls during successful memory encoding. The authors concluded that this finding reflected the role of a compensatory mechanism evoked in order to counteract the incipient AD-related neuropathology prior to the development of clinical dementia.

In another recent study, Hamalainen et al. [27] reported that individuals with MCI demonstrated increased fMRI activation in the posterior hippocampus, parahippocampus and fusiform gyrus in the presence of greater structural atrophy of the anterior portions of the hippocampus relative to normal control participants when performing an associative encoding task of novel picture-word pairs. In addition, the correlation between hippocampal volume and parahippocampal volume was negative in $\mathrm{MCI}$, but not in control or $\mathrm{AD}$ groups. The authors concluded that the increased activation in posterior medial temporal and fusiform regions was a compensatory mechanism related to anterior medial temporal lobe atrophy in MCI.

\section{Difficulties with Interpretation of fMRI Findings as Compensatory Neural Recruitment}

The noninvasive nature and relatively high temporal and spatial resolution of BOLD fMRI have made it an essential tool for studies of the working human brain. Most 
fMRI studies - including our own previous work - treat the BOLD response as an indirect qualitative measure of neural activity and interpret BOLD signal differences as differences in neural activity. However, the BOLD signal reflects local changes in deoxyhemoglobin content, which in turn exhibits a complex dependence on changes in cerebral blood flow (CBF), cerebral blood volume and the cerebral metabolic rate of oxygen consumption $\left(\mathrm{CMRO}_{2}\right)$ [28]. Of these quantities, $\mathrm{CMRO}_{2}$ is thought to be most tightly linked to neural activity, reflecting the fact that neurons necessarily expend energy to accomplish their work and that $\mathrm{CMRO}_{2}$ change reflects both synaptic and spiking activity $[29,30]$. The positive BOLD response observed in most fMRI experiments reflects the fact that CBF increases relatively more than $\mathrm{CMRO}_{2}$, so that local capillary and venous blood are more oxygenated during increased brain activity. In general, the actual amplitude of the BOLD response reflects a delicate balance between the relative increases in $\mathrm{CBF}$ and $\mathrm{CMRO}_{2}$. For these reasons, measures of $\mathrm{CBF}$ and $\mathrm{CMRO}_{2}$ changes are likely to provide a more accurate reflection of neural function than BOLD alone.

Factors that affect the baseline deoxyhemoglobin content (which we refer to as the proportionality constant $\mathrm{M}$ ) or the coupling between $\mathrm{CBF}$ and $\mathrm{CMRO}_{2}$ (which we refer to as the ratio $\mathrm{n}=\mathrm{CBF} / \mathrm{CMRO}_{2}$ ) may therefore alter the BOLD response even when neural activity is unchanged [for discussion, see ref. 31]. For example, there is growing evidence that changes in the cerebrovascular system due to age or disease can significantly alter the BOLD signal and complicate its interpretation [32]. Age-related factors include altered cerebrovascular ultrastructure, reduced elasticity of vessels, increased atherosclerosis, reduced resting-state CBF, decreased resting $\mathrm{CMRO}_{2}$, and reduced vascular reactivity to chemical modulators [32-37]. In fMRI studies of aging, researchers have found a significant age-related decrease in BOLD signal amplitude $[38,39]$, possibly reflecting age-related decreases in the elasticity of the cerebrovascular system $[32,40]$. Unfortunately, most fMRI studies have not investigated these additional variables of interest in order to take apart the neurovascular underpinnings of the BOLD signal, nor have a number of studies reported specific data on the structural volumes of their samples. In the next section, we provide recommendations for the incorporation of specific variables into functional neuroimaging studies to achieve a more comprehensive examination of the compensatory recruitment hypothesis.

\section{The RAP Model and Future Studies}

Working from a behavioral perspective, Dixon et al. [41] defined a compensatory mechanism as 'a cluster of behaviors and processes that are designed to overcome or mitigate cognitive deficits or declines'. Working from a functional neuroimaging perspective, and in response to the previously reviewed literature, it is clear that the definition of Dixon et al. needs to be further refined to include considerations of (1) brain region; (2) activation patterns, and (3) behavioral performance differences. We propose a modification of the definition by Dixon et al. of a compensatory mechanism to read: A cluster of behaviors and processes that are designed to overcome or mitigate cognitive deficits or declines [41] as manifested through either (1) additional brain regions, and/or (2) discrepant brain activation patterns, such that a cognitive ability is either (3) maintained or improved.

Also, functional neuroimaging studies interpreting their results as reflective of compensatory mechanisms have shown heterogeneity in populations of interest, imaging techniques, and numerous other methodological factors. As Aine et al. [42] suggest, methods used to examine age-related changes have limitations including use of the subtraction method, differences in resting state physiology, and differences in neurovascular coupling. Given this heterogeneity across studies, we further operationalize our proposed definition of compensatory mechanism recruitment by proposing a set of criteria to be considered when assessing whether a study's results are reflective of compensation. These guidelines have been developed with our three major considerations (region, activation, performance) and older adults in mind, but several of these points may be applicable to younger individuals as well.

\section{Whole-Brain Scans}

In view of our first major consideration of brain region, we specifically advocate the incorporation of volumetric information. Since recruitment of compensatory mechanisms often involves interactions between brain regions anatomically distant from each other [e.g. ref. 31], whole-brain scans or scans which encompass the majority of accessible gray matter will be important to consider in a functional paradigm. It is unlikely that reductions in asymmetry or other age-related effects will be observed unless they are explicitly tested [7]. Pattern of activation depends in part on region assessed (pathological processes associated with normal aging varies depending on particular region). For instance, neurofibrillary tangles may 
begin developing in the 30 s and 40 s and most adults accumulate them by age 60 [43]. There is a pattern that occurs with aging in which tangles first appear in the hippocampus and adjacent medial temporal structures and then affect frontal and cingulate areas followed by occipital and parietal areas [43]. Thus, although analyses involving specific regions of interest are useful, wholebrain analyses should also be considered in order to detect additional regions that may play a role in a potential compensatory mechanism. One useful methodology for achieving this aim involves creating a region of interest (ROI) that is homologous in both hemispheres and then calculating a lateralization index [7]. For instance, Cabeza [7] suggests calculating (right ROI - left ROI)/(right ROI + left ROI) and then multiplying the value by 100 . In such a case, a value of -100 indicates complete left lateralization of activation, 0 indicates perfect symmetry, and +100 indicates complete right lateralization. The derived lateralization index reflects whether a certain group is more or less lateralized than a comparison group. Of course, knowledge of normal or expected asymmetries in brain structure must be factored into such approaches. For example, the right hippocampus is normally larger than the left hippocampus, and a reduction in the expected asymmetry instead may represent a pathological marker [44].

Whole-brain functional scans also allow for the possible implementation of functional connectivity analyses. These statistical approaches identify regions of the brain that appear to be functionally associated in their activity, and they arguably are crucial for the determination of whether a compensatory network of regions is recruited during a particular task. Examples of significant contributions of functional connectivity analyses to compensation aging research include work from Cabeza et al. [45] and Daselaar et al. [46]. Cabeza et al. [45] utilized functional connectivity analyses to support the notion that older adults invoke more of a bilateral PFC activation pattern when compared to younger adults during a memory task, and Daselaar et al. [46] presented evidence supporting the notion that older adults may compensate for hippocampal decrements in functionality by recruiting rhinal cortex and bilateral prefrontal cortical regions.

\section{Segmentation Measurements}

It is also reasonable to assume that observed differences in activation patterns may be driven by any structural differences that may exist between groups. In the case of aging, structural deterioration may play a role in the functional recruitment of compensatory mechanisms
[47]. Segmentation measures are encouraged to be reported to account for this possibility. Issues related to structural integrity are thus critical to consider when studying age- or disease-related changes. Volumetric analyses and correction for cortical atrophy are important for assessing the functional significance of anatomical damage. In addition to examining the integrity of gray matter, assessing white matter integrity is important as white matter damage is relatively common in older adults [e.g. ref. 48], and may relate to alterations in CBF or cerebrovascular responsivity [49]. As Aine et al. [42] note, when examining age-related differences in functional neuroanatomy, many groups do not consider the plastic nature of the brain that is influenced by development and life experience through childhood and adulthood [50,51]. Studies of aging should consider both maturational and degenerative changes that occur during adulthood and aging, and as Aine et al. [42] suggest, brain maturation (e.g., myelination) continues into middle age 'when maximal WM volume and myelination are reached in frontal lobes and association areas, regions necessary for higher cognitive functions' [see also ref. 52]. In addition, postmortem evidence suggests that myelination of the hippocampus continues into the " 5 th and 6 th decades of life' [53]. In fact, both Aine et al. [42] and Raz et al. [54] have reported an inverted U-shaped function for white matter volume in frontal cortex in which young and older adults have reduced volumes relative to middle-aged adults. It is possible that older adults utilize a different strategy than the young adults in part as a result of different maturational stages.

\section{Activation Comparisons}

In our second major aspect of the RAP model, we specifically advocate the incorporation of activation comparisons and perfusion measurements. The interpretation of compensatory neural recruitment is predicted on the ability to discern its absence or presence within a controlled investigation. This can be accomplished by the use of two or more subject groups and statistical comparisons between the groups, or through the use of temporal analyses that associate the presence of a compensatory mechanism during periods of time when such a mechanism may be needed and the absence of such a mechanism during periods of time when it is not. The pattern of optimal response should be carefully considered. In another study, Cook et al. [55] reported that on a delayed matchto-sample task, older adults displayed larger regions of activation as well as greater interindividual variability regarding extent of activation. Analyses yielded mixed age- 
related differences in connectivity, with older adults showing both less and more connectivity, depending on the network. Thus, it may be necessary to consider activation, deactivation, connectivity patterns (using functional connectivity analyses), and specific additional regions when considering optimal activation pattern response.

\section{Perfusion Measurements}

It is also worth noting that fMRI studies of normal older adults and at-risk groups have reported inconsistent findings at times (e.g., increased versus decreased brain response). As we previously noted [23], this may be related to uncertainties about the mechanisms linking the hemodynamic response and underlying neuroanatomy and neurophysiology. Studies have shown age-related changes in the hemodynamic response $[19,32,38]$. Our recent work has revealed at least three different hemodynamic scenarios that could cause perceived 'compensatory' increases in fMRI activity. The first scenario is an increased BOLD response due to a true compensatory increase in neural activity reflected by a normal $\mathrm{CBF} /$ $\mathrm{CMRO}_{2}$ coupling ratio $\mathrm{n}$, as depicted in figure 1a. However, a second scenario that could lead to this perception is an increase in the CBF response (perhaps caused by increased cerebrovascular disease), but the neural activity and $\mathrm{CMRO}_{2}$ changes are maintained, thereby increasing $\mathrm{n}$. This second scenario is depicted in figure $1 \mathrm{~b}$. A third scenario is one in which baseline $\mathrm{CBF}$ is reduced while baseline $\mathrm{CMRO}_{2}$ activity, neural activity and the neurovascular coupling ratio $\mathrm{n}$ remain constant. This causes an increase in the proportionality constant (M), leading to increased BOLD response, which is depicted in figure 1c. Thus, an identical increase in the BOLD response can arise in three very different ways. As the figure illustrates, a method to clarify which one of these scenarios may be occurring is possible with the addition of perfusion measurements and cerebral blood volume mapping, both of which may similarly help to adjust for baseline differences in signal intensity.

\section{Explicit Cognitive Task Control}

Finally, in view of our third major consideration for the RAP model (i.e. behavioral performance), we specifically advocate explicit cognitive task control. The design of the cognitive task should control for level of performance, which may involve titration of difficulty or ensuring equality of performance between groups. It is important to have similar performances between groups so that activation differences cannot be attributed to other factors such as effort. Moreover, it is problematic to compare
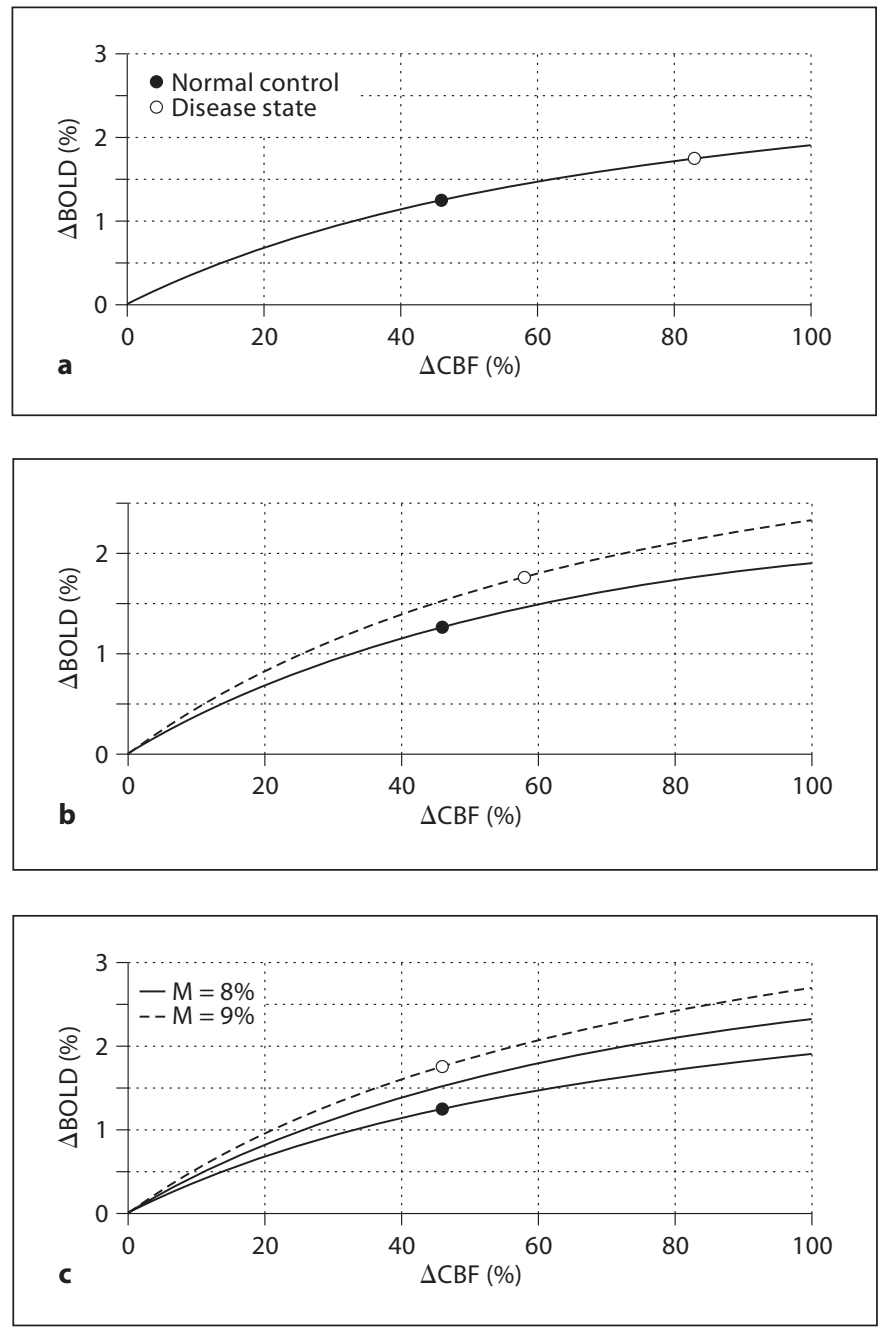

Fig. 1. Three physiological scenarios leading to a 'compensatory' increase in the BOLD response, each showing a $1.25 \%$ BOLD response in the 'normal' condition and a $1.75 \%$ BOLD response in the 'compensatory' condition. In this example, the normal condition has the proportionality constant $\mathrm{M}=8 \%$ and the neurovascular coupling $\left(\mathrm{n}=\mathrm{CBF} / \mathrm{CMRO}_{2}\right)$ at $\mathrm{n}=3$. a An increased BOLD response due to a true compensatory increase in neural activity and a normal $\mathrm{CBF} / \mathrm{CMRO}_{2}$ coupling ratio $\mathrm{n}$. $\mathbf{b}$ The $\mathrm{CBF}$ response is increased (perhaps by increased stroke risk), but the neural activity and $\mathrm{CMRO}_{2}$ changes are maintained, thereby increasing $\mathrm{n}$. c Baseline CBF is reduced while baseline $\mathrm{CMRO}_{2}$ activity, neural activity, and $n$ remain constant. This causes an increase in $M$, leading to increased BOLD response. Thus an identical increase in the BOLD response can arise in three very different ways. Adapted from Brown et al. [60], with permission.

difficulty across different tasks because difficulty may be confounded with different cognitive processes used to complete tasks (e.g. verbal and arithmetic tasks) so it is ideal to use the same task with varying levels of difficulty. 
There may be an 'upper limit' to the brain's ability to engage compensatory mechanisms characterized by easier levels of a task showing compensation/increased activation whereas more difficult levels would outstrip the brain's ability to compensate. If there are at least three levels of difficulty, the association between brain activation and cognitive demand can be more thoroughly probed, and nonlinear relations could be detected. Average performance across various conditions or levels can be analyzed and subjective ratings of task difficulty or effort collected.

Another possible approach involves using the simplest version of a task so that all individuals perform well [42]. As Nielson et al. [56] suggest, it must be clear whether there is a direct benefit of increased activation in order to consider activation as compensatory. Compensation should result in improved performance or decreased impairment. Even if a brain region is active during a certain task, it does not necessarily imply that this region is necessary to complete this task or is playing a compensatory role [50].

\section{Concluding Remarks}

We conclude with three final considerations when interpreting functional neuroimaging results as compensatory neural recruitment. First, quantitative data should be considered in addition to qualitative data and relative differences. As Gould et al. [57] note, it is unclear whether compensation involves qualitative or quantitative changes or both. In fact, Stern [58] presented a model in which greater response of regions (i.e. quantitative changes) underlying a task in healthy adults reflects cognitive reserve, and recruitment of additional regions not typically underlying the task (i.e. qualitative changes) represents compensation. Importantly, implicit in the quantitative-change hypothesis is a linear relationship between BOLD activation and increased cognitive load, which may or may not be the case.

Second, qualitatively different patterns of brain activity may reflect use of different strategies to complete cognitive tasks. For instance, Aine et al. [42] propose that the divergent pattern of response in older subjects in many studies may reflect the role of subvocalizations or inner speech in facilitating memory processes. As Aine et al. [42] note, during the verbal delayed-matching-to-sample task, the older adults demonstrated greater signal amplitudes in areas including the dorsolateral prefrontal cortex and anterior cingulate. However, as the young-adult group was not fully matured (as evidenced by the finding that middle-aged adults had greater white matter volumes compared to both the young and older adults), the different brain responses between young and older adults may reflect learned strategies related to normal maturation rather than compensation. It is important to consider a variety of potential explanations of both quantitative and qualitative differences.

Third, there are a variety of demographic variables that are essential to consider when interpreting age-related changes in brain activation. Functional neuroimaging studies have compared relatively small groups of individuals and there is little information regarding the generalizability of these findings to broader populations. It is important to evaluate cognitive functioning as it affects brain activity. Age-related changes in activation may be specific to those individuals who are experiencing cognitive decline. This decline may be extremely subtle so it is important to screen participants carefully. For instance, education may influence the relation between cognition and effects on the brain. In addition, gender may influence age-related changes on the brain and influence lateralization [7]. Longitudinal studies may also be useful although they have the disadvantage that older adults demonstrate less change in brain activation related to practice effects compared to younger adults [59].

Interest in compensatory neural recruitment has grown with the advent of functional neuroimaging techniques and recent advances in aging research. The utility of a standardized set of criteria for interpreting results as evidence for compensatory mechanism recruitment would be significant given this rapidly growing body of literature. We propose our RAP model as one possible set of criteria for investigators to consider when developing future programs of research designed to investigate these important mechanisms using fMRI approaches. While our proposed set of criteria was developed specifically with fMRI in mind, we could foresee aspects of this model as applicable to other functional neuroimaging methods as well (e.g., PET, SPECT).

\section{Acknowledgements}

This critical review was supported by funds from National Institutes of Health grants F31 NS059193 (K.J.B.), K24 AG026431 (M.W.B.), P50 AG005131 (M.W.B.), RO1 AG012674 (M.W.B.), and by grant IIRG-07-59343 (M.W.B.) from the Alzheimer's Association. 


\section{References}

-1 Han SD, Houston WS, Jak AJ, Eyler LT, Nagel BJ, Fleisher AS, Brown GG, Corey-Bloom J, Salmon DP, Thal LJ, Bondi MW: Verbal paired-associate learning by APOE genotype in non-demented older adults: fMRI evidence of a right hemisphere compensatory response. Neurobiol Aging 2007;28: 238-247.

2 Li SC, Lindenberger U: Cross-level unification: a computational exploration of the link between deterioration or cognitive abilities in old age; in Nilsson LG, Markowitsch HJ (eds): Cognitive Neuroscience of Memory. Seattle, Hogrefe \& Huber, 1999, pp 103-146.

3 Welford AT: Performance, biological mechanisms and age: a theoretical sketch; inWelford AT, Birren JE (eds): Behavior, Aging, and the Nervous System. Springfield, Thomas, 1965, pp 3-20.

$\checkmark 4$ Salthouse T: Initiating the formalization of theories of cognitive aging. Psychol Aging 1988;3:3-16.

5 Cabeza R, Grady CL, Nyberg L, McIntosh AR, Tulving E, Kapur S, et al: Age-related differences in neural activity during memory encoding and retrieval: a positron emission tomography study. J Neurosci 1997;17: 391-400.

6 Grady CL, Bernstein LJ, Beig S, Siegenthaler AL: The effects of encoding task on age-related differences in the functional neuroanatomy of face memory. Psychol Aging 2002;17:17-23.

7 Cabeza R: Hemispheric asymmetry reduction in older adults: the HAROLD model. Psychol Aging 2002;17:85-100.

$\checkmark 8$ Reuter-Lorenz PA, Jonides J, Smith ES, Hartley A, Miller A, Marshuetz C, et al: Age differences in the frontal lateralization of verbal and spatial working memory revealed by PET. J Cogn Neurosci 2000;12:174-187.

$\checkmark 9$ Cabeza R, Anderson ND, Locantore JK, McIntosh AR: Aging gracefully: compensatory brain activity in high-performing older adults. NeuroImage 2002;17:1394-1402.

$\checkmark 10$ Anderson KE, Perera GM, Hilton J, Zubin N, Dela Paz R, Stern Y: Functional magnetic resonance imaging study of word recognition in normal elders. Prog Neuropsychopharmacol Biol Psychiatry 2002;26:647650

-11 Grady CL, Maisog JM, Horwitz B, Ungerleider LG, Mentis MJ, Salerno JA, et al: Agerelated changes in cortical blood flow activation during visual processing of faces and location. J Neurosci 1994;14:1450-1462.

- 12 Davis SW, Dennis NA, Daselaar SM, Fleck MS, Cabeza R: Que PASA? The posterior-anterior shift in aging. Cereb Cortex 2008;18: 1201-1209.

13 Beason-Held LL, Kraut MA, Resnick SM: II. Temporal patterns of longitudinal change in aging brain function. Neurobiol Aging 2008; 29:497-513.
14 Li SC, Lindenberger U, Sikstrom S: Aging cognition: from neuromodulation to representation. Trends Cogn Sci 2001;5:479-486.

15 Iidaka T, Sadato N, Yamada H, Murata T, Omori M, Yonekura Y: An fMRI study of the functional neuroanatomy of picture encoding in younger and older adults. Cogn Brain Res 2001;11:1-11.

16 Erickson KI, Colcombe SJ, Wadhwa R, Bherer L, Peterson MS, Scalf PE, Kim JS, Alvarado M, Kramer AF: Training-induced plasticity in older adults: effects of training on hemispheric asymmetry. Neurobiol Aging 2007;28:272-283.

17 Colombe SJ, Kramer AF, Erickson KI, Scalf P: The implications of cortical recruitment and brain morphology for individual differences in inhibitory function in aging humans. Psychol Aging 2005;20:363-375.

18 Cabeza R: Cognitive neuroscience of aging: Contributions of functional neuroimaging. Scand J Psychol 2001;42:277-286.

19 D’Esposito M, Zarahn E, Aguirre GK, Rypma B: The effect of normal aging on the coupling of neural activity to the BOLD hemodynamic response. NeuroImage 1999;10: 6-14.

$>20$ Li SC, Sikstrom S: Integrative neurocomputational perspectives on cognitive aging, neuromodulation, and representation. Neurosci Biobehav Rec 2002;26:795-808.

21 Bookheimer SY, Strojwas MH, Cohen MS, Saunders AM, Pericak-Vance MA, Mazziotta JC, Small GW: Patterns of brain activation in people at risk for Alzheimer's disease. N Engl J Med 2000;343:450-456.

22 Cohen JD, Forman SD, Braver TS, Casey BJ, Servan-Schrieber D, Noll DC: Activation of the prefrontal cortex in a nonspatial working memory task with functional MRI. Hum Brain Mapp 1994;1:293-304.

23 Bondi MW, Houston WS, Eyler LT, Brown GG: FMRI evidence of compensatory mechanisms in older adults at genetic risk for Alzheimer disease. Neurology 2005;64:501508.

24 Burggren AC, Small GW, Sabb FW, Bookheimer SY: Specificity of brain activation patterns in people at genetic risk for Alzheimer disease. Am J Geriatr Psychiatry 2002;10:44-51.

25 Dickerson BC, Salat DH, Bates JF, et al: Medial temporal lobe function and structure in mild cognitive impairment. Ann Neuro 2004;56:27-35.

26 Kircher TT, Weis S, Freymann K, Erb M, Jessen F, Grodd W, Heun R, Leube DT: Hippocampal activation in patients with mild cognitive impairment is necessary for successful memory encoding. J Neurol Neurosurg Psychiatry 2007;78:812-818.
27 Hamalainen A, Pihlajamaki M, Tanila H, Hanninen T, Niskanen E, Tervo S, Karjalainen PA, Vanninen RL, Soininen H: Increased fMRI responses during encoding in mild cognitive impairment. Neurobiol Aging 2007;28:1889-1903.

$>28$ Buxton RB, Uludag K, Dubowitz DJ, Liu TT: Modeling the hemodynamic response to brain activation. NeuroImage 2004;23(suppl 1):S220-S233.

29 Attwell D, Laughlin SB: An energy budget for signaling in the grey matter of the brain. J Cereb Blood Flow Metab 2001;21:11331145 .

30 Hyder F: Neuroimaging with calibrated fMRI. Stroke 2004;35(11 suppl 1):26352641.

31 Davis TL, Kwong KK, Weisskoff RM, Rosen BR: Calibrated functional MRI: mapping the dynamics of oxidative metabolism. Proc Natl Acad Sci USA 1998;95:1834-1839.

>32 D’Esposito M, Deouell LY, Gazzaley A: Alterations in the BOLD fMRI signal with ageing and disease: a challenge for neuroimaging. Nat Rev Neurosci 2003;4:863-872.

33 Bentourkia M, Bol A, Ivanoiu A, Labar D, Sibomana M, Coppens A, Michel C, Cosnard G, De Volder AG: Comparison of regional cerebral blood flow and glucose metabolism in the normal brain: effect of aging. J Neurol Sci 2000;181:19-28.

34 Claus JJ, Breteler MM, Hasan D, Krenning EP, Bots ML, Grobbee DE, Van Swieten JC, Van Harskamp F, Hofman A: Regional cerebral blood flow and cerebrovascular risk factors in the elderly population. Neurobiol Aging 1998;19:57-64.

35 Kawamura J, Terayama Y, Takashima S, Obara K, Pavol MA, Meyer JS, Mortel KF, Weather S: Leuko-araiosis and cerebral perfusion in normal aging. Exp Aging Res 1993; 19:225-240.

\$6 Markus H, Cullinane M: Severely impaired cerebrovascular reactivity predicts stroke and TIA risk in patients with carotid artery stenosis and occlusion. Brain 2001;124(pt 3):457-467.

37 Yamaguchi T, Kanno I, Uemura K, Shishido F, Inugami A, Ogawa T, Murakami M, Suzuki K: Reduction in regional cerebral metabolic rate of oxygen during human aging. Stroke 1986;17:1220-1228.

>38 Buckner RL, Snyder AZ, Sanders AL, Raichle ME, Morris JE: Functional brain imaging of young, nondemented, and demented older adults. J Cog Neurosci 2000;12(suppl 2):2434.

39 Tekes A, Mohamed MA, Browner NM, Calhoun VD, Yousem DM: Effect of age on visuomotor functional MR imaging. Acad Radiol 2005; 12:739-745. 
-40 Uspenskaia O, Liedetrau M, Herms J, Danek A, Hamann GF: Aging is associated with increased collagen type IV accumulation in the basal lamina of human cerebral microvessels. BMC Neurosci 2004;5:37.

-41 Dixon RA, Hopp GA, Cohen A-L, de Frias CM, Backman L: Self-reported memory compensation: similar patterns in Alzheimer's disease and very old adult samples. J Clin Exp Neuropsychol 2003;25:382-390.

-42 Aine CJ, Woodruff CC, Knoefel JE, Adair JC, Hudson D, Qualls C, Bockholt J, Best E, Kovacevic S, Cobb W, Padilla D, Hart B, Stephen JM: Aging: Compensation or maturation? NeuroImage 2006;32:1891-1904.

$\checkmark 43$ Braak H, Braak E: Frequency stages of Alzheimer-related lesions in different age categories. Neurobiol Aging 1997;18:351357.

44 Jak AJ, Houston WS, Nagel BJ, Corey-Bloom J, Bondi MW: Differential cross-sectional and longitudinal impact of APOE genotype on hippocampal volumes in nondemented older adults. Dement Geriatr Cogn Disord 2007;23:382-389.

-45 Cabeza R, McIntosh AR, Grady CL, Nyberg L, Houle S, Tulving, E: Age-related changes in neural interactions during memory encoding and retrieval: A network analysis of PET data. Brain Cogn 1997;35:369-372.

-46 Daselaar SM, Prince SE, Cabeza R: When less means more: deactivations during encoding that predict subsequent memory. NeuroImage 2004;23:921-927.
47 Hayes SM, Cabeza R: Imaging aging: present and future; in Hofer SM, Alwin DF (eds) Handbook of Cognitive Aging: Interdisciplinary Perspectives. Thousand Oaks, Sage, 2008, pp 308-326.

48 Fazekas F, Schmidt R, Kleinert R, et al: The spectrum of age-associated brain abnormalities: their measurement and histopathological correlates. J Neural Transm Suppl 1998, 53:31-39.

49 Bangen KJ, Restom K, Liu TT, Jak AJ, Perthen JE, Wierenga CE, Salmon DP, Bondi MW Differential age effects on cerebral blood flow and BOLD response to encoding: associations with cognition and stroke risk. Neurobiol Aging 2007, Epub ahead of print.

50 Poldrack RA: Imaging brain plasticity: conceptual and methodological issues - A theoretical review. NeuroImage 2000;12:1-13.

-51 Schlaggar BL, Brown TT, Lugar HM, Visscher KM, Miezin FM, Petersen SE: Functional neuroanatomical differences between adults and school-age children in the processing of single words. Science 2002;296: 1476-1479.

52 Bartzokis G: Schizophrenia breakdown in the well-regulated lifelong process of brain development and maturation. Neuropsychopharmacology 2002;27:672-683.

53 Benes FM, Turtle M, Khan Y, Farol P: Myelination of a key relay zone in the hippocampal formation occurs in the human brain during childhood, adolescence, and adulthood. Arch Gen Psychiatry 1994;51:477484 .
54 Raz N, Lindenberger U, Rodrigue KM, Kennedy KM, Head D, Williamson A, Dahle C, Gerstorf D, Acker JD: Regional brain changes in aging healthy adults: general trends, individual differences and modifiers. Cereb Cortex 2005; 15:1676-1689.

55 Cook IA, Bookheimer S, Mickes L, Leuchter AF, Kumar A: Aging and brain activation with working memory tasks: an fMRI study of connectivity. Int J Geriatr Psychiatry 2007; 22:332-342.

56 Nielson KA, Langenecker SA, Garavan HP: Differences in the functional neuroanatomy of inhibitory control across the adult life span. Psychol Aging 2002;17:56-71.

57 Gould RL, Arroyo B, Brown RG, Owen AM, Bullmore ET, Howard RJ: Brain mechanisms of successful compensation during learning in Alzheimer disease. Neurology 2006;67: 1011-1017.

58 Stern Y: What is cognitive reserve? Theory and research application of the reserve concept. J Int Neuropsychol Soc 2002;8:448460.

59 Madden DJ, Turkington TG, Provenzale JM, Denny LL, Hawk TC, Gottlob LT, et al: Adult age differences in the functional neuroanatomy of verbal recognition memory. Hum Brain Mapp 1999;7:115-135.

60 Brown GG, Perthen JE, Liu TT, Buxton RB: A primer on functional magnetic resonance imaging. Neuropsychol Rev 2007;17:107125 . 